\section{Traitement expérimental de la trypanosomiase africaine par le facteur trypanolytique humain combiné à un nanocorps}

Toya Nath Baral, Stefan Magez, Benoît Stijlemans, Katja Conrath, Benoit Vanhollebeke, Etienne Pays, Serge Muyldermans, Patrick De Baetselier

> La trypanosomiase africaine humaine, ou maladie du sommeil, résulte d'une infection par des protozoaires parasites appelés trypanosomes, qui sont transmis d'hôte à hôte par des glossines hématophages généralement dénommées mouches tsé-tsé. La sous-espèce Trypanosoma brucei rhodesiense est responsable d'une forme aiguë de la maladie en Afrique de l'Est. Cette maladie est fatale si elle n'est pas traitée à temps. Les trypanosomes échappent au système immunitaire de l'hôte grâce à un mécanisme sophistiqué de variation antigénique [1] qui leur permet de changer continuellement l'antigène majeur, le VSG (variant surface glycoprotein), qui couvre la totalité de leur surface. Ce processus de variation laisse peu d'espoir d'éliminer le parasite par vaccination. Par ailleurs, les médicaments actuels, suramine et mélarsoprol, sont toxiques et peu efficaces étant donné l'acquisition de résistance par le parasite [2]. Par conséquent, le développement de nouveaux médicaments, plus spécifiques et moins toxiques, est nécessaire.

On sait depuis longtemps que le sérum humain normal tue la plupart des trypanosomes, à l'exception de ceux responsables de la maladie du sommeil. Dans le cas de $T$. b. rhodesiense, la résistance à la lyse par le sérum humain est due à une seule protéine appelée SRA (serum resistance-associated) [3]. Cette protéine interagit fortement et spécifi- quement avec une lipoprotéine appelée apolipoprotéine L-I (APOLI), qui s'est révélée être le facteur trypanolytique du sérum humain [4]. L'APOLl contient trois domaines, un domaine formateur de pores ioniques similaire à celui des colicines bactériennes, un domaine dont la structure est sensible au $\mathrm{pH}$ et qui est capable d'installer la protéine dans diverses membranes biologiques, et enfin un domaine interactif carboxyterminal, qui est reconnu par SRA (pour revue récente, voir [5]). Cette lipoprotéine, qui est associée à une sous-fraction des HDL, est capturée par endocytose par le trypanosome. En raison de l'acidification consécutive au processus de digestion, un changement conformationnel permet à l'APOLl de se détacher des particules d'HDL et de s'insérer dans la membrane lysosomiale où l'activité formatrice de pores entraîne un flux irréversible d'ions chlorure du cytoplasme vers le lysosome. Le flux ionique, associé à un passage d'eau, provoque un gonflement osmotique incontrôlable de la vacuole digestive, entraînant la mort du parasite [5]. Seuls les domaines formateurs de pores et d'adressage membranaire sont nécessaires à l'activité trypanolytique de I'APOLl. Par conséquent, la lipoprotéine dépourvue du domaine interagissant avec SRA (Tr-APOLl) est toujours trypanolytique et, surtout, devient capable de tuer également $T$. $b$. rhodesiense car elle n'est plus neutralisable par SRA [4].
T.N. Baral, S. Magez, B. Stijlemans, K. Conrath, S. Muyldermans, P. De Baetselier : Département des Interactions cellulaires et moléculaires, Vlaams Interuniversitair Instituut voor Biotechnologie, Laboratorium voor Cellulaire en Moleculaire Immunologie, Vrije Universiteit Brussel, Pleinlaan 2, B-1050 Bruxelles, Belgique.

B. Vanhollebeke, દ. Pays :

Laboratoire de Parasitologie moléculaire, IBMM, Université Libre de Bruxelles,

12 , rue des Professeurs Jeener et Brachet, B-6041 Gosselies, Belgique.

tbaral@vub.ac.be

Tr-APOLl représente donc un nouveau médicament actif contre la trypanosomiase humaine, à condition que la compétition avec I'APOLl endogène $(10 \mu \mathrm{g} / \mathrm{ml})$ soit évitée. Cela requiert le ciblage sélectif de Tr-APOLl sur le parasite.

Les nanocorps ( $\mathrm{Nb}$, nanobodies) sont des polypeptides à simple domaine dérivés d'anticorps propres aux camélidés (chameaux, lamas), qui sont capables de se fixer sur des antigènes avec une haute affinité $[6,7]$. Étant donné leur petite taille (13 kDa) par rapport au Fab, le domaine équivalent des anticorps classiques ( $55 \mathrm{kDa}$ ), et leur comportement strictement monomérique, ces polypeptides représentent des outils idéaux pour cibler la surface du parasite. Des nanocorps reconnaissant spécifiquement cette surface ont été sélectionnés par immunisation de chameaux avec des préparations purifiées d'antigènes de $T$. brucei, et criblage des anticorps de ces chameaux par expression de bibliothèques d'ADNc sous forme d'affichage en phage display confronté à des VSG de différents clones et sous-espèces de T. brucei. 
Le critère de sélection était la reconnaissance d'épitopes invariants. En effet, étant donné leur petite taille, les nanocorps peuvent détecter des épitopes qui sont cryptiques pour les anticorps conventionnels, ou même pour les fragments Fab dérivés de ces anticorps, et qui seraient conservés indépendamment de la variation antigénique. C'est ainsi qu'un nanocorps, désigné NbAn33, fut sélectionné sur la base de sa reconnaissance d'un épitope constant de VSG [8] (Figure IA). $\mathrm{NbAn} 33$ reconnaît spécifiquement le radical oligomannose, car il s'attache à des résidus Man9 et Man7 synthétiques, se fixe de façon indifférente à divers VSG qui ne partagent que le radical $M_{\text {5-9 }}$ attaché à une asparagine, et ne reconnaît plus le VSG dépourvu de ce radical. Par conséquent, NbAn33 a été utilisé pour la production de différentes chimères Nb-Tr-APOLl [9]. Un conjugué dont le nanocorps était espacé de Tr-APOLl par la charnière naturelle d'anticorps $\gamma 2 c$ de lama (Figure 1B), s'est révélé capable de se fixer spécifiquement à la surface du trypanosome,

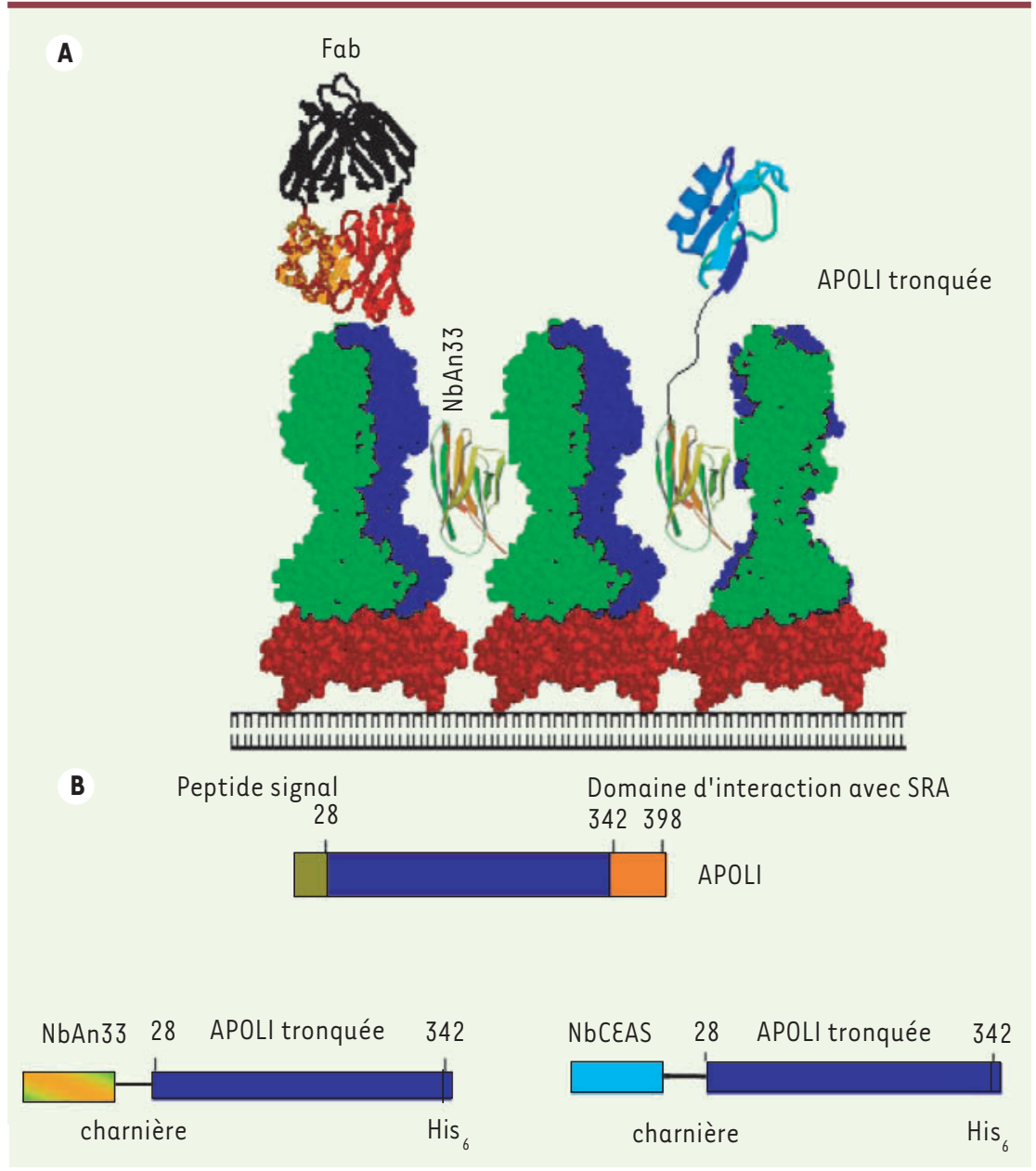

Figure 1. A. La surface du trypanosome est couverte par un manteau dense et variable de VSG, rendant les épitopes invariables sous-jacents inaccessibles pour de grandes molécules telles que le fragment Fab des anticorps classiques, présents chez la majorité des vertébrés. Les nanocorps de chameaux, tels que NbAn33, même couplés à une molécule toxique, peuvent atteindre le radical oligomannose présent chez différents VSG. B. La protéine SRA, qui interagit avec le domaine carboxy-terminal du facteur trypanolytique humain, I'APOLl, rend $T$. b. rhodesiense résistant au sérum humain. L'APOLI tronquée, dépourvue de domaine interagissant avec SRA, a été fusionnée avec NbAN33 ou avec un nanocorps contrôle (NbCEA5). sans reconnaître de façon détectable différentes cellules de l'hôte comme les globules rouges ou des suspensions cellulaires de foie, de nodules lymphoïdes ou de rate de souris infectées. In vitro, NbAn33-Tr-APOLl a manifesté une activité trypanolytique très supérieure à celle d'un conjugué contrôle, où le nanocorps utilisé (NbCEA5) reconnaissait un épitope absent de la surface du parasite. In vivo, NbAn33Tr-APOLl s'est révélé capable de guérir la trypanosomiase expérimentale chez la souris. Des souris infectées avec des souches virulentes de T. b. rhodesiense, puis traitées par une seule injection intrapéritonéale de NbAn33-Tr-APOLl, sont débarrassées complètement et durablement du parasite sans manifester de symptômes liés au traitement (Figure 2). Des résultats similaires furent observés dans des modèles d'infection plus chronique (trypanosomes pléomorphes, capables de se différencier en formes quiescentes appelées short stumpy). Dans ce cas, l'injection intrapéritonéale de NbAn33-Tr-APOLl après (plutôt qu'avant) le premier pic de parasitémie - a permis d'éliminer les parasites pour une longue période, mais l'infection a repris en raison de la survie de parasites dans le foie. La période sans parasites fut néanmoins totalement débarrassée des signes pathologiques liés à l'infection, comme l'anémie, la perte de poids corporel, le gonflement de la rate, les lésions hépatiques et la perte d'activité locomotrice.

En conclusion, la version tronquée de I'APOLl combinée à un nanocorps capable de cibler la surface du parasite constitue une nouvelle molécule trypanocide potentiellement utilisable pour traiter la maladie du sommeil humaine, soit seule, soit en combinaison avec des doses sous-curatives d'autres médicaments. On peut s'attendre à ce que NbAn33-Tr-APOLl n'induise pas ou peu de réponse immunitaire humorale chez l'homme, car I'APOLl est un antigène naturel humain, et la 

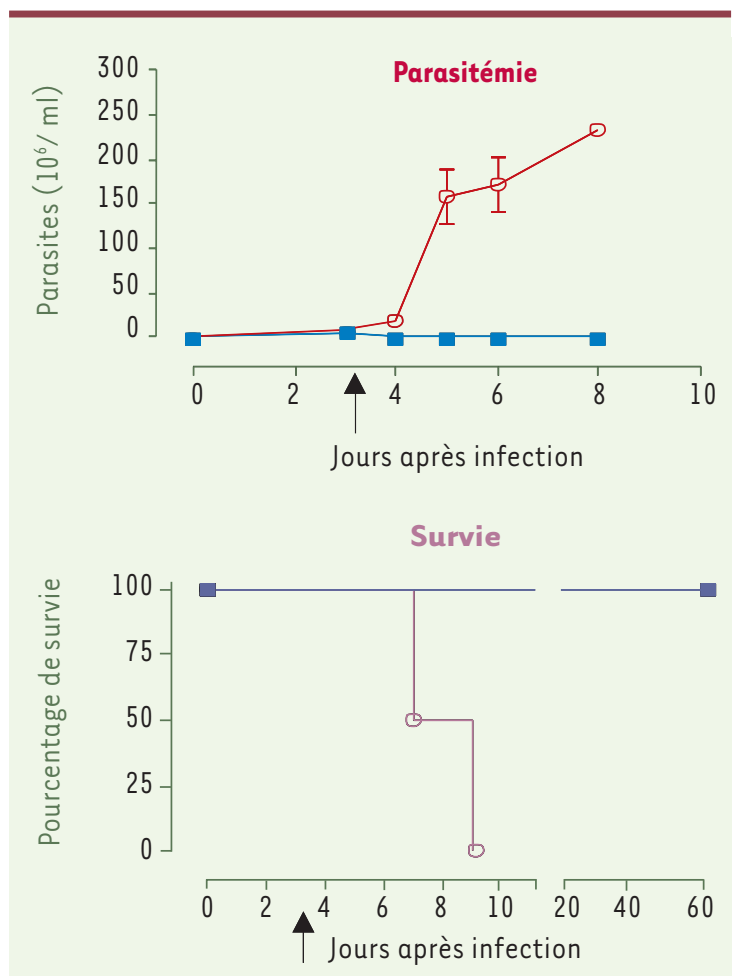

Figure 2. Des souris infectées par T.b. rhodesiense ont été traitées au jour 3 par injection intrapéritonéale (flèche) de NbAn33-Tr-APOL1 (courbe bleue) ou de NbCEA5-Tr-APOL1 (courbe rouge). Le ciblage spécifique de l'APOLI tronquée via le nanocorps élimine les parasites, et les souris survivent au moins jusqu'à la fin de l'expérience, tandis qu'à la dose utilisée l'APOLI non ciblée ne bloque pas l'infection. séquence des nanocorps est très semblable à celle de domaines variables d'immunoglobulines humaines. Si nécessaire, cette séquence pourrait d'ailleurs être «humanisée» davantage [10]. D'autres développements sont possibles, car la substitution de NbAn33 par NbES31, un nanocorps dirigé contre la sous-unité ESAG6 du récepteur trypanosomal de la transferrine, s'est révélée aussi efficace pour éliminer les trypanosomes chez des souris infectées. Enfin, il n'y a aucune raison que la stratégie de ciblage grâce à des nanocorps ne puisse être appliquée à d'autres agents infectieux, en fonction de la disponibilité de nanocorps spécifiques et de molécules naturelles de défense de l'hôte. $\diamond$
Experimental therapy of African trypanosomiasis with a nanobodyconjugated human trypanolytic factor

\section{RÉFÉRENCES}

1. Pays $\varepsilon$, Vanhamme L, Perez-Morga D. Antigenic variation in Trypanosoma brucei: facts, challenges and mysteries. Curr Opin Microbiol $2004 ; 7$ : 369-74.

2. Legros D, Ollivier G, Gastellu-Etchegorry $M$, et al. Treatment of human African trypanosomiasis : present situation and needs for research and development. Lancet Infect Dis $2002 ; 2$ : 437-40.

3. Xong HV, Vanhamme L, Chamekh M, et al. A VSG expression site-associated gene confers resistance to human serum in Trypanosoma rhodesiense. Cell 1998 ; $95: 839-46$.

4. Vanhamme L, Paturiaux-Hanocq F, Poelvoorde P, et al. Apolipoprotein L-I is the trypanosome lytic factor of human serum. Nature $2003 ; 422: 83-7$.

5. Pays $\varepsilon$, Vanhollebeke B, Vanhamme L, et al. The trypanolytic factor of human serum. Nat Rev Microbiol $2006 ; 4: 477-86$.

6. Muyldermans $\mathrm{S}$. Single domain camel antibodies: current status. J Biotechnol $2001 ; 74: 277-302$.

7. Hamers-Casterman C, Atarhouch T, Muyldermans S, et al. Naturally occurring antibodies devoid of light chains. Nature 1993 ; 363 : 446-8.

8. Stijlemans B, Conrath K, Cortez-Retamozo V, et al Efficient targeting of conserved cryptic epitopes of infectious agents by single domain antibodies. African trypanosomes as paradigm. J Biol Chem 2004 ; 279: 1256-61.

9. Baral TN, Magez S, Stijlemans B, et al. Experimental therapy of African trypanosomiasis with a nanobodyconjugated human trypanolytic factor. Nat Med 2006 ; $12: 580-4$.

10. Conrath K, Vincke C, Stijlemans B, et al. Antigen binding and solubility effects upon the veneering of a camel VHH in framework-2 to mimic a VH.J Mol Biol $2005 ; 350: 112-25$

\section{NOUVELLE}

\section{PCSK9, du gène à la protéine Un nouvel acteur dans I'homéostasie du cholestérol}

Marianne Abifadel, Jean-Pierre Rabès, Catherine Boileau, Mathilde Varret

> L'hypercholestérolémie familiale, à transmission autosomique dominante $(A D H)$, est l'une des maladies génétiques les plus fréquentes (1/500 sous sa forme hétérozygote). L'excès de cholestérol lié aux lipoprotéines de basse densité (LDL, low density lipoproteins) provoque des dépôts artériels à l'origine de plaques d'athérome, sources de complications cardiovasculaires graves. L'ADH est due à des mutations des gènes codant le récepteur des LDL ( $L D L R)$ ou son ligand l'apolipoprotéine $B-100$ ( $A P O B)$. Nous avons identifié le troisième gène impliqué dans l'ADH, PCSK9, localisé
M. Abifadel : Inserm U781, Paris, France ; AP-HP, Hôpital Necker-Enfants Malades, 149, rue de Sèvres, 75743 Paris, France ; Université Paris 5, Faculté de médecine, Paris, France ; Université Saint-Joseph, Faculté de Pharmacie, BP11, 5076 Beyrouth, Liban.

J.P. Rabès, C. Boileau : Inserm U781, 75743 Paris, France ;

Université Paris 5, 75005 Paris, France ;

AP-HP, Hôpital Ambroise Paré, Laboratoire de Biochimie et de Génétique Moléculaire ;

Université Versailles Saint-Quentin-en-Yvelines, UFR Médicale Paris Ile-de-France Ouest,

92104 Boulogne, France.

M. Varret: Inserm U781, 75743 Paris, France ;

Université Paris 5, 75005 Paris, France.

abifadel@necker.fr 